Arch. Vet. Scienc., 4(1):41-44, 1999

Printed in Brazil

\title{
O CLOPROSTENOL E O HORMÔNIO LIBERADOR DE GONADOTROFINAS NA PERFORMANCE REPRODUTIVA DE VACAS LEITEIRAS APÓS O PUERPÉRIO
}

\author{
LUIZ ERNANDES KOZICKI; ${ }^{1}$; ROMILDO ROMUALDO WEISS ${ }^{1}$ \\ ${ }^{1}$ Departamento de Medicina Veterinária, Setor de Ciências Agrárias, UFPR, C.P. 2959, CEP 80 035-050, \\ Curitiba - PR. - Brasil.
}

\begin{abstract}
The purpose of the present research work was to study estrus' induction after the puerperal period in Holstein dairy cows. A herd of 50 Holstein dairy cows from the Curitiba's region were used in this experiment. They were divided in three groups. At the 50th day post partum, Cloprostenol, Gonadorelin $=$ $\mathrm{GnRH}=$ and saline solution were administered by intramuscular injection to group $1(\mathrm{n}=24), 0.5 \mathrm{mg}$ of Cloprostenol, to group $2(\mathrm{n}=14), 250 \mu \mathrm{g}$ of $\mathrm{GnRH}$, and to group $3(\mathrm{n}=12), 5 \mathrm{ml}$ of saline as placebo. Rectal palpation was performed in all animals on the $42 / 43 \mathrm{rd}$, the $50 \mathrm{th}=$ the day of the intramuscular injection $=$ and on the 57th and 64th day post partum, in order to know the uterus condition and the ovary's functions. It has been found that Cloprostenol induces cow's estrus more quickly than GnRH or the placebo $(\mathrm{p}<0.01)$. The rate of pregnancy after the $1 \mathrm{st}$ and the 2nd artificial insemination, under Cloprostenol effect (84.5\%) was greater than under $\mathrm{GnRH}(75 \%)$ or the placebo $(67 \%)$ at the control group. The parturition/pregnancy interval in the Cloprostenol group was smaller (89 days) than in the GnRH (127.1 days) or in the control (108.8 days) groups. The number of animals that did not respond to the treatment was smaller in the Cloprostenol group. On the other hand, the GnRH group was more efficient $(\mathrm{p}<0.05)$ than the control group on the estrus' induction $(18.6$ versus 43.5 days, respectively). The GnRH and the Cloprostenol groups displayed higher rates of silent estrus (33.3 and $28.5 \%$, respectively) than the control group $(16.6 \%)(\mathrm{p}<0.05)$.
\end{abstract}

RESUMO - O propósito da presente pesquisa foi o de estudar a indução do estro após o período puerperal em vacas de leite Holstein. Um grupo de 50 vacas da raça Holstein, oriundas da região de Curitiba, foi utilizado neste experimento. Para tanto, esses animais foram divididos em três grupos. No $50^{\circ}$ dia após o parto, Cloprostenol, Gonadorelina $=\mathrm{GnRH}=$ e soro fisiológico foram administrados por injeção intramuscular na seguinte ordem: ao grupo $1(\mathrm{n}=24), 0.5$ mgde Cloprostenol, ao grupo $2(\mathrm{n}=14), 250 \mu \mathrm{g}$ de $\mathrm{GnRH}$, e ao grupo $3(\mathrm{n}=12)$ - grupo controle - $5 \mathrm{ml}$ de soro fisiológico. Palpação retal foi levada a efeito em todos os animais no $42^{\circ} / 43^{\circ}$, no $50^{\circ}=$ dia da administração das substãncias em estudo $=$ e no $57^{\circ}$ e no $64^{\circ}$ dias post partum, a fim de conhecer as condições do útero e das funções ovarianas. Verificou-se que o Cloprostenol induz o estro more rapidamente do que o GnRH ou o placebo $(\mathrm{p}<0.01)$. O índice de prenhez após a primeira e a segunda inseminações artificiais, quando sob a ação do Cloprostenol $(84.5 \%)$ foi superior àquele que ocorreu com o GnRH (75\%) ou com o placebo (67\%), neste caso no grupo controle. O intervalo parto/prenhez no grupo do Cloprostenol foi menor (89 dias) doque no caso do GnRH (127.1 dias) ou do grupo controle (108.8 days). O número de animais que não responderam aos diferentes tratamentos foi menor no caso do grupo do Cloprostenol. Por outro lado o tratamento com GnRH mostrou-se mais eficiente $(\mathrm{p}<0.05)$ do que o grupo controle com respeito à indução ao estro (18.6 e 43.5 dias, respectivamente). Os grupos do GnRH e do Cloprostenol revelaram índices maiores de estro silencioso (33.3 e $28.5 \%$, respectivamente), diferente do que ocorreu com o grupo controle $(16.6 \%)(\mathrm{p}<0.05)$.

\section{Introdução}

Vacas com elevada produção leiteira tendem a demonstrar o estro somente após o segundo mês depois do parto, acarretando perda de vida reprodutiva do animal. Para reduzir esse tempo, pesquisadores passaram a utilizar técnicas de indução do estro mediante agentes luteolíticos como a prostaglandina $\mathrm{F}_{2} \alpha \quad\left(\mathrm{PGF}_{2} \alpha\right)$ ou seu análogo, o Cloprostenol, bem como com hormonios liberadores de gonadotrofinas (GnRH).

Pesquisa conduzida por ETHERINGTON et al. (1984) visando melhoria da fertilidade dos bovinos a partir da terceira semana pós-parto, dá conta de que esses pesquisadores obtiveram o intervalo parto-primeiro estro visível e o intervalo parto- concepção no $55^{\circ}$ e $109^{\circ}$ dias respectivamente. Outros trabalhos a esse respeito foram executados, dentre os quais destacam-se os de SAMBA (1985), SHCHEGLOVA e D'YAKONOW (1986), MODIANO(1987), KLINSKII et al. (1988) e de OKUDA et al. (1988). OKUDA et al. (1988) administraram $\mathrm{PGF}_{2} \alpha$ a um grupo de vacas no $47^{\circ}$ dia post partum (p.p.). Os resultados evidenciaram que os animais tratados tiveram o intervalo partoconcepção menor (78,7 dias) do que o dos controles (109,1 dias), embora não diferindo quanto à taxa de prenhez entre os grupos. Semelhantes resultados foram constatados por KLINSKII et. al (1988) ao utilizarem o agente luteolítico.

Com referência ao uso do GnRH, FONSECA et al. (1980) haviam relatado que o uso do hormônio 
liberador de gonado trofina restabelecia o estro precocemente após o parto, quando utilizado em rebanhos bem manejados. KRAL et al. (1984) utilizaram GnRH entre o $12^{\circ}$ e o $33^{\circ}$ dia post partum obtendo resultados de intervalo partoinseminação artificial de 67,7 e 69,2 dias para os grupos tratado e controle respectivamente. Esses autores verificaram também o intervalo partoprenhez de 86,9 e 96,2 dias com taxa de concepção na $1^{\text {a }}$ inseminação artificial de 48,1 e $45,7 \%$ para os grupos tratado e não-tratados respectivamente. KOZICKI (1991), tratando vacas leiteiras com GnRH no $35^{\circ}$ dia pós-parto visando a indução ao estro, evidenciou essa característica em $32,3 \%$ no lote tratado e apenas $7,2 \%$ no controle, obtendo índice de ovulação no grupo tratado de 73,4\% contra 37,7 \% no controle. KUDLAC et al. (1985), tratando vacas após o $60^{\circ}$ dia pós-parto com GnRH conseguiram taxa de prenhez de $73,3 \%$ contra $53,0 \%$ nos controles.

Face aos discrepantes resultados das pesquisas relatadas, é proposição do presente trabalho realizar estudo comparativo entre o Cloprostenol, um análogo da $P G F \alpha_{2}$, e a Gonadorelina, GnRH, observando-se a eficiência de cada um e seus efeitos quanto aos parâmetros reprodutivos em vacas leiteiras da Região Metropolitana de Curitiba.

\section{Material e Métodos}

Foram utilizadas vacas leiteiras da raça Holandesa Preta e Branca, procedentes da Região Metropolitana de Curitiba, PR. A alimentação dos animais baseou-se em silagem de milho, concentrado na quantidade dependente da produção de cada animal e forragens de inverno e verão fornecidas ad libitum. Os dados dos animais foram protocolados em fichas individuais, segundo os achados dos exames ginecológicos de palpação retal. As vacas foram divididas em três grupos: Grupo 1 constituído por 24 animais que no $50^{\circ}$ dia pós-parto apresentaram corpo lúteo em um dos ovários. Nesses animais administrou-se uma dose via intramuscular de $0,5 \mathrm{mg}$ de Cloprostenol (Ciosin, Coopers do Brasil) para indução ao estro e na observação deste realizava-se inseminação artificial (IA). O segundo grupo de vacas foi composto por 14 animais que no $50^{\circ}$ dia pós-parto não detinham corpo lúteo nos ovários. Nesses animais, aplicou-se $250 \mu \mathrm{g}$ de Gonadorelina (Profertil, Tortuga Cia Zootécnica) via intramuscular com a finalidade de indução ao estro. Igualmente, na observação deste, os animais eram inseminados. O terceiro grupo de vacas constituiuse de 12 animais (grupo controle) que recebeu intramuscularmente $5 \mathrm{ml}$ de solução fisiológica estéril como placebo. Todos os animais foram palpados via retal nos seguintes dias após o parto: $42^{\circ} / 43^{\circ}, 50^{\circ}$ (dia do tratamento), $57^{\circ}$ e $64^{\circ}$ dia. Pelo toque retal foram verificadas as condições do útero dos animais (grossura de cornos, simetria e contratilidade), bem como os ovários nos seus aspectos de funcionalidade (presença de folículo, corpo lúteo, cistos, aciclia) Os exames ginecológicos seguiram critérios adotados por GRUNERT (1988). O tratamento estatístico baseou-se na média, desvio padrão, teste $\mathrm{T}$ de Student e do $\mathrm{X}^{2}$.

\section{Resultados e Discussão}

Os resultados estão expostos nas Tabelas 1, 2 e 3. Pela análise da variável intervalo partotratamento, verifica-se que embora as visitas às propriedades tenham sido realizadas uma vez semanalmente, torna-se possível equacionar esse fato, uma vez que a média para todos os grupos foi idêntica, com baixo desvio padrão.

Tabela 1. Parâmetros de idade, tipo de parto, expulsão de placenta, sexo do bezerro e número de partos de vacas leiteiras tratadas com GnRH e Cloprostenol. (Curitiba, PR) $(n=50)$.

\begin{tabular}{l|c|c|c|c|c|c|c|c}
\hline \multicolumn{1}{c|}{ Grupos } & Idade & \multicolumn{2}{c|}{ Parto } & \multicolumn{2}{c|}{ Placenta } & \multicolumn{2}{c}{ Bezerro } \\
& $\begin{array}{c}\text { (meses) } \\
(\mathrm{x} \pm \mathrm{s})\end{array}$ & $\begin{array}{c}\text { Normal } \\
\%\end{array}$ & $\begin{array}{c}\text { Difícil } \\
\%\end{array}$ & $\begin{array}{c}\mathrm{R} \\
\%\end{array}$ & $\begin{array}{c}\text { NR } \\
\%\end{array}$ & $\begin{array}{c}\mathrm{M} \\
\%\end{array}$ & $\begin{array}{c}\mathrm{F} \\
\%\end{array}$ & $\begin{array}{c}\left(\mathrm{n}^{\mathrm{o}}\right) \\
(\mathrm{x} \pm \mathrm{s})\end{array}$ \\
\hline GnRH & $52,5 \pm 22,2$ & 83,3 & 16,7 & 20,9 & 78,1 & $54,1^{\mathrm{e}}$ & 45,9 & $2,5 \pm 1,5$ \\
Cloprostenol & $46,5 \pm 11,6$ & $100^{\mathrm{a}}$ & & & $100^{\mathrm{c}}$ & $78,6^{\mathrm{f}}$ & 21,4 \\
Controle & $46,2 \pm 37,2$ & $67,0^{\mathrm{b}}$ & 37,0 & 25,0 & $75,0^{\mathrm{d}}$ & 61,5 & 38,5 \\
\hline
\end{tabular}

$a: b=p<0,05 c: d=p>0,05$ e:f= p $>0,05 R=$ retenção; $N R=$ não retenção; $M=$ macho; $F=$ fêmea.

Para melhorar a fertilidade em bovinos, ETHERINGTON et al. (1984) relataram o uso do agente luteolítico a partir da terceira semana pósparto, obtendo intervalo parto-primeiro estro visível no $55^{\circ}$ dia e o intervalo parto-concepção no $110^{\circ}$ dia. O efeito luteolítico do Cloprostenol verificado na presente pesquisa corrobora os dados dos autores supracitados, pois o estro visível fez-se presente também no $54^{\circ}$ dia após o parto. A involução do corpo lúteo ocorreu 4,8 dias após sua aplicação e atingiu $92,3 \%$ dos animais. Os animais tratados com Cloprostenol demonstraram o estro e foram 
Cloprostenol e GnRH na Reprodução de Vacas Leiteiras após o Puerpério

inseminados em tempo significativamente mais curto do que os grupos GnRH e controle (Tabela 2). As vacas que receberam cloprostenol acusaram, no $88^{\circ}$ dia após o parto, $84,5 \%$ de prenhez, ao passo que os lotes de animais que injetados com gonadorelina e solução fisiológica alcançaram índices respectivamente de $75 \%$ e $67 \%$ de prenhez no $127^{\circ}$ e $108^{\circ}$ dia após o parto. Não houve significância estatística na variável taxa de prenhez entre os três grupos, apesar da vantagem em prol do análogo da $\mathrm{PGF}_{2} \alpha$, considerando-se a $1^{\mathrm{a}}$ e a $2^{\mathrm{a}}$ inseminações. Esse fato é corroborado por OKUDA et al. (1988), os quais trataram vacas no $47^{\circ}$ dia pós-parto com cloprostenol e evidenciaram intervalo parto-concepção bastante vantajoso frente aos controles.

Tabela 2. Parâmetros reprodutivos de intervalo parto-tratamento, tratamento-estro, tratamento- $1^{\mathrm{a}} \cdot 2^{\mathrm{a}}$ IA, bem como o índice de prenhez na $1^{\mathrm{a}}$ e $2^{\mathrm{a}}$ IA em vacas leiteiras tratadas com $\mathrm{GnRH}$ e Cloprostenol. (Curitiba, PR) $(\mathrm{n}=50)$.

\begin{tabular}{|c|c|c|c|c|c|c|}
\hline \multirow[b]{2}{*}{ Grupos } & \multicolumn{4}{|c|}{ Intervalo(dias) } & \multicolumn{2}{|c|}{ Índice de prenhez } \\
\hline & $\begin{array}{c}\text { Parto/tratamento } \\
(\mathrm{x} \pm \mathrm{s})\end{array}$ & $\begin{array}{c}\text { Tratamento/estro } \\
(\mathrm{x} \pm \mathrm{s})\end{array}$ & $\begin{array}{c}\text { Tratamento/ } 1^{a} I A \\
(x \pm s)\end{array}$ & $\begin{array}{c}\text { Tratamento/2a } \\
(\mathrm{x} \pm \mathrm{s})\end{array}$ & $\begin{array}{c}1^{\mathrm{a}} \mathrm{IA} \\
\%\end{array}$ & $\begin{array}{c}2^{\mathrm{a}} \mathrm{IA} \\
\%\end{array}$ \\
\hline $\begin{array}{c}\text { GnRH } \\
\text { Cloprostenol } \\
\text { Controle }\end{array}$ & $\begin{array}{l}48,2 \pm 1,9 \\
49,0 \pm 1,9 \\
48,6 \pm 3,3\end{array}$ & $\begin{array}{c}18,6 \pm 10,8^{\mathrm{a}} \\
4,8 \pm 2,8^{\mathrm{b}} \\
43,5 \pm 29,8^{\mathrm{c}}\end{array}$ & $\begin{array}{c}18,6 \pm 10,8 \\
4,8 \pm 3,0 \\
43,5 \pm 29,8\end{array}$ & $\begin{array}{l}77,1 \pm 17,1^{\text {d }} \\
39,0 \pm 15,3^{\text {e }} \\
58,8 \pm 12,4^{\text {f }}\end{array}$ & $\begin{array}{c}45 \\
30,7 \\
42\end{array}$ & $\begin{array}{c}30 \\
53,8 \\
25\end{array}$ \\
\hline
\end{tabular}

$a: b=p<0,01 \quad a: c=p<0,05 \quad b: c=p<0,01 \quad d: e=p<0,05 d: f=p>0,05$. IA = Inseminação artificial.

Ao se confrontar em dados do grupo GnRH e os do controle, observa-se que o primeiro acusou significância $(\mathrm{p}<0,05)$ nas variáveis intervalo tratamento-estro e tratamento-primeira inseminação artificial. KRAL et al. (1984) utilizaram GnRH no puerpério obtendo resultados de intervalo partoprenhez de 86,9 a 96,2 dias com taxa de prenhez na primeira inseminação artificial de 48,1 e 45,7\% para os grupos tratado e controle respectivamente. Na comparação, os resultados da presente pesquisa mostraram-se melhores quanto à precocidade do estro (66,8 dias) frente aos dos autores acima mencionados, sem diferenças, porém quanto à taxa de prenhez.

Neste trabalho observou-se, igualmente, o percentual de estro silencioso nos animais (Tabela 3). Embora sem registro de significância estatística entre os grupos, os animais que receberam GnRH demonstraram percentual mais elevado $(33,3 \%)$ que os demais $(28,5$ e $16,6 \%$ respectivamente para os grupos Cloprostenol e controle).

Tabela 3. Dados de estro silencioso em vacas leiteiras após administração de GnRH e Cloprostenol no período pós puerperal. (Curitiba, PR) (n=50).

\begin{tabular}{l|c|c}
\multicolumn{1}{c|}{ Grupos } & Estro silencioso & $\%$ \\
\hline GnRH & $8 / 24$ & $33,3^{\mathrm{a}}$ \\
Cloprostenol & $4 / 14$ & 28,5 \\
Controle & $2 / 12$ & $16,6^{\mathrm{b}}$ \\
\hline
\end{tabular}

$\mathrm{a}: \mathrm{b}=\mathrm{p}>0,05$

Assim, pelos resultados obtidos pode-se concluir que o Cloprostenol mostrou-se significativamente mais eficiente no que diz respeito à indução do estro, do que os grupos GnRH e controle $(\mathrm{p}<0,01)$, mostrando índice de prenhez mais elevado $(80,5 \%)$ nas duas primeiras inseminações artificiais quando comparado com os grupos $\mathrm{GnRH}(75 \%)$ e controle (67\%) revelando ainda menor intervalo partoconcepção (89 dias) do que o grupo GnRH (127,1 dias) e controle (108,8 dias). Verificou-se ainda que o número de animais que não respondeu ao tratamento com o Cloprostenol foi menor do que os outros grupos. Por outro lado o grupo $\mathrm{GnRH}$ mostrou-se mais eficiente $(p<0,05)$ do que o grupo controle quanto à rapidez de induzir o estro bem como taxa de prenhez mais elevada na primeira e na segunda inseminações artificiais muito embora o intervalo parto-concepção tenha sido mais elevado.

\section{REFERENCIAS BIBLIOGRÁFICAS}

ETHERINGTON, W.G.; BOSU, W.T.; MARTIN, S.W.;COTE, J.F.; DOIG, P.A; LESLIE, K.E. Reproductive performance in dairy cows following postpartum treatment with $\mathrm{GnRH}$ and/or prostaglandin. A field trial. In 10th INTERNATIONAL CONGRESS ON ANIMAL REPRODUCTION AND ARTIFICIAL INSEMINATION. University of Illinois, Brief Communications, v.III, 1984.

FONSECA, F.A.; BRITT, J.H.; KOSUGIYAMA, M.; RITCHIE, H.D.; DILLARD, E.U. Ovulation, ovarian function and reproductive performance after treatment with GnRH in postpartum suckled cows. Theriogenology, 13(2):171-181, 1980.

GRUNERT, E. Examenes en el marco del control de fertilidad. In: GRUNERT,E. \& BERCHTOLD, M. Infertilidad en la vaca. Hemisferio Sur, $1^{\text {a }}$ ed. Uruguai, 1988, pg. 55-88. 
KLINSKII, Y.D.;SKEIKIN, V.N.;DEVOV, Y.M. Comparative avaluation of oestrophan cloprostenol and soprostone. Zootekhniya, 4:50-51, 1988.

KOZICKI, L.E. Contribuição ao tratamento do anestro pós-puerperal em vacas leiteiras. Rev.Set.Cien.Agr. 10(1-2):157-162,1991

KRAL, J.; SEVCIK,B.; BILEK, P.; BOROVICKA, A.; STROKOVA, J; PICMAUSOVA, D.; KROUPOVA, $\mathrm{V}$. The possibility of using gonadorelin (Dirigestran Spofa) in controlling reproductive functions in cattle. Biologizace a Chemizace Zivicisne Vyroby, Veterinaria, 20(6):505-516, 1984

KUDLAC, E.; VRTEL, M.; VLCEK, Z.; VINKLEP, A Stimulation of conception in cows using GnRH at spontaneous oestrus. Biologizace e Chemizace Zivocisne Vyroby, Veterinaria, 21(1):5-13,1985.
OKUDA, K.; GAONA, W.A.; SATO, K. Effects of gonadotropin releasing hormone and prostaglandin F2 alpha on the reproductive performance in post partum cow. Theriogenology, 29(4):823-833, 1988.

MODIANO, R.I. Actividad ovarica posparto e dos hatos de ganado Holstein en Aguascalientes. Efecto de la prostaglandina F2 alfa sobre algunos parametros reproductivos. Veterinaria, 18(1):91-92, 1987.

SAMBA, D. The postpartum use of oestrophan on cows with inadequate cycling and hypofunctional ovaries. Intesif.Proizvodstva moloka i govyadiny, 60-63, 1985.

SHCHEGLOVA, N.; D'YAKONOV, E.F Synchronization of oestrus in cows. Vosproizvodstvo Sel.Kloz. zhivotnykh i kriokonservatsiya gamet i embrionov, 20-21, 1986. 